\title{
Dielectrophoretic force and torque on a sphere in an arbitrary time varying electric field
}

\author{
C. Y. Yang \\ Department of Automatic Control Engineering, Chungchou Institute of Technology, Yuan Lin, \\ Changhua 51003, Taiwan, Republic of China \\ U. Lei $^{\mathrm{a})}$ \\ Institute of Applied Mechanics, National Taiwan University, Taipei 10764, Taiwan, Republic of China
}

(Received 18 August 2006; accepted 7 September 2006; published online 20 October 2006)

\begin{abstract}
The dielectrophoretic force and torque on a sphere were derived in an arbitrary time varying electric field using the effective moment method. The results depend on the local instantaneous electric field felt by the particle and the polarization history experienced by the particle. The polarization history effect is significant for a moving particle when it is traveling with a sufficiently high speed, when it is traveling across a nonuniform field with a sufficiently small length scale, or when the applied electric frequency is relatively low. The results may find application in the ac electrokinetic manipulation of particles and cells. (C) 2006 American Institute of Physics.
\end{abstract}

[DOI: 10.1063/1.2364468]

Dielectrophoresis is an effective tool in the manipulation of particles in microfluidic devices generated by microelectrodes. ${ }^{1,2}$ As a particle moves during the manipulation, it cuts across the spatial nonuniform field (required for dielectrophoresis) with varying velocities, and is exposed to an arbitrary time varying electric field and experiences unsteady viscous drag and resistive torque from the surrounding fluid in general. The goal of the present work is to study the effect of the time varying field on the induced effective dipole moment, and thus the dielectrophoretic force and torque, of a spherical particle.

Denegri et al. ${ }^{3}$ had derived the dielectrophoretic force and torque on a spherical particle in an arbitrary time varying electric field in terms of infinite series using the Maxwell stress tensor method (see also Ref. 4). The results may also be derived rather easily using the effective moment method, and the analysis for the leading order is shown as follows.

For currents and frequencies typically used in dielectrophoresis, we may employ the quasistatic Maxwell's equations, ${ }^{5}$ and the equation for electric potential, $\Phi$, can be reduced to

$$
\sigma \nabla^{2} \Phi+\varepsilon \frac{\partial}{\partial t} \nabla^{2} \Phi=0
$$

for a homogeneous linear dielectric with permittivity $\varepsilon$ and conductivity $\sigma$. Consider a sphere with radius $R$ in an arbitrary time varying electric field

$$
\mathbf{E}=-\nabla \Phi=E_{x}(t) \hat{e_{x}},
$$

where $E_{x}(t)$ is an arbitrary function of time, $t$, and $\hat{e_{x}}$ is the unit vector along a given $x$ axis. The electric potential outside $\left(\Phi_{1}\right)$ and that inside $\left(\Phi_{2}\right)$ the sphere are governed by Eq. (1) and are solved subjected to Eq. (2) together with the boundary conditions on the surface of the sphere, which include the continuity of the potential,

$$
\Phi_{1}=\Phi_{2} \quad \text { at } \quad r=R,
$$

and the instantaneous charge conservation,

\footnotetext{
a) Author to whom correspondence should be addressed; electronic mail: leiu@spring.iam.ntu.edu.tw
}

$$
\sigma_{1} E_{r 1}-\sigma_{2} E_{r 2}+\frac{\partial \sigma_{f}}{\partial t}=0 \quad \text { at } \quad r=R
$$

where the free (unpaired) electric surface charge $\sigma_{f}=\varepsilon_{1} E_{r 1}$ $-\varepsilon_{2} E_{r 2}$, and $E_{r 1}=-\partial \Phi_{1} / \partial r$ and $E_{r 2}=-\partial \Phi_{2} / \partial r$ are the radial components of the electric field. Throughout this letter, the subscripts " 1 " and " 2 " refer to the properties of the materials outside and inside the sphere, respectively. In the above equations, we have employed spherical coordinates $(r, \theta, \psi)$ fixed at the center of the sphere, with $\theta=0$ coincide with the $x$ direction in Eq. (2). For such a geometric arrangement, the potentials $\Phi_{1}$ and $\Phi_{2}$ are independent of $\psi$.

By taking the Laplace transform of Eq. (1), and assuming that $\Phi=0$ when $t \leqslant 0$, we have

$$
(\sigma+\varepsilon s) \nabla^{2} \widetilde{\Phi}=0,
$$

where $\widetilde{\Phi}(r, \theta ; s)=\int_{0}^{\infty} e^{-s t} \Phi(r, \theta ; t) d t$ is the Laplace transform of $\Phi$. The solutions for $\nabla^{2} \widetilde{\Phi}_{1}=0$ and $\nabla^{2} \widetilde{\Phi}_{2}=0$ satisfying Eq. (2) are available in Ref. 1, page 10. By applying the boundary conditions at $r=R$ in the Laplace domain, which are obtained by taking the Laplace transform of Eqs. (3a) and (3b), the particular solution can be obtained. In particular, the magnitude of the induced effective dipole moment ${ }^{1}$ corresponding to the electric field in Eq. (2) in the Laplace domain is

$$
\begin{aligned}
m_{x}(s) & =4 \pi \varepsilon_{1} A(s) \\
& =4 \pi \varepsilon_{1} R^{3} E_{x}(s) \frac{\varepsilon_{2}-\varepsilon_{1}}{\varepsilon_{2}+2 \varepsilon_{1}}\left[1+\frac{\tau_{0}^{-1}-\tau_{\mathrm{MW}}^{-1}}{\tau_{\mathrm{MW}}^{-1}+s}\right] .
\end{aligned}
$$

Here $\tau_{0}=\left(\varepsilon_{2}-\varepsilon_{1}\right) /\left(\sigma_{2}-\sigma_{1}\right)$ and $\tau_{\mathrm{MW}}=\left(\varepsilon_{2}+2 \varepsilon_{1}\right) /\left(\sigma_{2}+2 \sigma_{1}\right)$. $\tau_{0}$ is of unit of time, and may be positive or negative depending on the relative magnitudes of the material parameters. $\tau_{\mathrm{MW}}$ is the relaxation time for Maxwell-Wagner surface polarization. By taking the inverse Laplace transformation of Eq. (5), we obtain 


$$
\begin{aligned}
m_{x}(t)= & 4 \pi \varepsilon_{1} R^{3} \frac{\varepsilon_{2}-\varepsilon_{1}}{\varepsilon_{2}+2 \varepsilon_{1}}\left\{E_{x}(t)+\left(\frac{1}{\tau_{0}}-\frac{1}{\tau_{\mathrm{MW}}}\right)\right. \\
& \left.\times \int_{0}^{t} E_{x}(\tau) \exp \left(-\frac{t-\tau}{\tau_{\mathrm{MW}}}\right) d \tau\right\} .
\end{aligned}
$$

If the electric field is $\mathbf{E}(t)=E_{x}(t) \hat{e_{x}}+E_{y}(t) \hat{e_{y}}+E_{z}(t) \hat{e_{z}}$ instead of Eq. (2), the induced effective dipole moment can be obtained via superposition as

$$
\begin{aligned}
\mathbf{m}(t)= & m_{x}(t) \hat{e_{x}}+m_{y}(t) \hat{e_{y}}+m_{z}(t) \hat{e_{z}} \\
= & 4 \pi \varepsilon_{1} R^{3} \frac{\varepsilon_{2}-\varepsilon_{1}}{\varepsilon_{2}+2 \varepsilon_{1}}\left\{\mathbf{E}(t)+\left(\frac{1}{\tau_{0}}-\frac{1}{\tau_{\mathrm{MW}}}\right)\right. \\
& \left.\times \int_{0}^{t} \mathbf{E}(\tau) \exp \left[-\frac{(t-\tau)}{\tau_{\mathrm{MW}}}\right] d \tau\right\} .
\end{aligned}
$$

When the particle is moving through a time and spatial varying electric field, it is more appropriate to express $\mathbf{E}(t)$ in Eq. (7) as $\mathbf{E}(\mathbf{Y}(t), t)$. Here $\mathbf{Y}(t)$ is the instantaneous location of the particle determined via Newton's second law of motion. The notation $\mathbf{E}(\mathbf{Y}(t), t)$ has the physical implication that the unsteadiness of the electric field felt by a moving particle has two origins: one is the temporal change of the field itself and the other is caused by the translation of the particle through a nonuniform electric field, and is named here the "crossing trajectory effect." Such a notation for the field felt by a moving particle has been employed widely in the area of twophase suspension flow. ${ }^{6}$

With Eq. (7), we can calculate the dielectrophoretic force $(\mathbf{F})$ and torque about a diameter of the sphere $(\mathbf{T})$ at time $t$ through the effective moment method. ${ }^{1}$ They are

$$
\begin{aligned}
\mathbf{F}(t)= & \mathbf{m} \cdot \nabla \mathbf{E} \\
= & 4 \pi \varepsilon_{1} R^{3} \frac{\varepsilon_{2}-\varepsilon_{1}}{\varepsilon_{2}+2 \varepsilon_{1}}[\mathbf{E}(\mathbf{Y}(t), t) \cdot \nabla \mathbf{E}(\mathbf{Y}(t), t) \\
& +\left(\frac{1}{\tau_{0}}-\frac{1}{\tau_{\mathrm{MW}}}\right) \int_{0}^{t} \mathbf{E}(\mathbf{Y}(\tau), \tau) \cdot \nabla \mathbf{E}(\mathbf{Y}(t), t) \\
& \left.\times \exp \left(-\frac{(t-\tau)}{\tau_{\mathrm{MW}}}\right) d \tau\right]
\end{aligned}
$$

and

$$
\begin{aligned}
\mathbf{T}(t)= & \mathbf{m} \times \mathbf{E} \\
= & 4 \pi \varepsilon_{1} R^{3} \frac{\varepsilon_{2}-\varepsilon_{1}}{\varepsilon_{2}+2 \varepsilon_{1}}\left[\left(\frac{1}{\tau_{0}}-\frac{1}{\tau_{\mathrm{MW}}}\right) \int_{0}^{t} \mathbf{E}(\mathbf{Y}(\tau), \tau)\right. \\
& \left.\times \mathbf{E}(\mathbf{Y}(t), t) \exp \left(-\frac{(t-\tau)}{\tau_{\mathrm{MW}}}\right) d \tau\right],
\end{aligned}
$$

respectively, which agree with the results of Denegri et al. ${ }^{3}$ after some manipulation. The first terms on the right hand side of Eqs. (6)-(8) are the quasistatic terms, which involved only the local instantaneous field felt by the particle. The second terms (the integral terms) are the polarization history terms, which depend on the history of the electric field experienced by the particle. For a time varying field on a particle without considering the crossing trajectory effect, a scaling analysis of Eqs. (6)-(8) shows that the ratio of the polarization history term to the quasistatic term is of order of Downloaded 19 Dec 2008 to 140.112.113.225. Redistribution subje
$\left(\tau_{\mathrm{MW}} / \tau_{0}-1\right)$. For a typical case with $\varepsilon_{1}=2.5 \varepsilon_{0}, \varepsilon_{2}=10 \varepsilon_{0}$, $\sigma_{1}=4 \times 10^{-8} \mathrm{~S} / \mathrm{m}$, and $\sigma_{2}=10^{-8} \mathrm{~S} / \mathrm{m}$ (Ref. 1, page 41), where $\varepsilon_{0}=8.854 \times 10^{-12} \mathrm{~F} / \mathrm{m}$ is the permittivity of free space, we found $\left(\tau_{\mathrm{MW}} / \tau_{0}-1\right)=-2.5$, which implies that the polarization history term and the quasistatic term are of the same order. Note also that there is no quasistatic term for the torque in Eq. (9).

The validity of Eq. (6) was also checked against the special cases in literatures, ${ }^{1}$ which include the transient response of an Ohmic dielectric sphere subjected to a suddenly applied dc electric field, the long time response of an Ohmic dielectric sphere subjected to a rotating field, and that subjected to an ac field. For the latter case, with $\mathbf{E}(t)$ $=E_{0} \cos (\omega t) \hat{e_{x}}$, we found from Eq. (6) that

$m_{x}(t)=4 \pi \varepsilon_{1} R^{3} E_{0}\{\operatorname{Re}[K(\omega)] \cos (\omega t)-\operatorname{Im}[K(\omega)] \sin (\omega t)\}$

after neglecting a transient exponential term, $-\operatorname{Im}[K(\omega)] \exp \left(-t / \tau_{\mathrm{MW}}\right)$. Here $E_{0}$ and $\omega$ are the amplitude and angular frequency of the applied field, and

$$
\begin{aligned}
\operatorname{Re}[K(\omega)]= & \frac{\varepsilon_{2}-\varepsilon_{1}}{\varepsilon_{2}+2 \varepsilon_{1}}+\left(\frac{\sigma_{2}-\sigma_{1}}{\sigma_{2}+2 \sigma_{1}}-\frac{\varepsilon_{2}-\varepsilon_{1}}{\varepsilon_{2}+2 \varepsilon_{1}}\right) \\
& \times \frac{1}{1+\omega^{2} \tau_{\mathrm{MW}}^{2}}
\end{aligned}
$$

and

$$
\operatorname{Im}[K(\omega)]=-\left(\frac{\sigma_{2}-\sigma_{1}}{\sigma_{2}+2 \sigma_{1}}-\frac{\varepsilon_{2}-\varepsilon_{1}}{\varepsilon_{2}+2 \varepsilon_{1}}\right) \frac{\tau_{\mathrm{MW}} \omega}{1+\omega^{2} \tau_{\mathrm{MW}}^{2}}
$$

are the real and imaginary parts, respectively, of the complex Clausius-Mossotti factor.

One interesting issue in the previous results is the crossing trajectory effect on the polarization history for a moving particle. Consider the traveling wave electric field generated by the "comb" geometries of the microelectrode system in Fig. 1(a) of Ref. 7. There are two parallel arrays of electrodes separated by a channel on a surface. The traveling wave electric field was established by sequentially addressing the electrodes with sinusoidal voltages of phase separation at $0^{\circ}$, $-90^{\circ},-180^{\circ}$, and $-270^{\circ}$ and keeping directly opposing electrodes on either sides of the channel phase shifted from each other by $-180^{\circ}$. Suppose a particle is translating with a constant velocity $V \hat{e_{Y}}$ along the central axis ( $Y$ axis) of the channel. The field felt by the translating particle can be expressed as

$$
\begin{aligned}
\mathbf{E} & =E_{0} \cos \left(\omega t+\frac{2 \pi Y}{\lambda}\right) \hat{e_{X}}=E_{0} \cos \left(\omega t+\frac{2 \pi V t}{\lambda}\right) \hat{e_{X}} \\
& \equiv E_{0} \cos \left(\omega^{\prime} t\right) \hat{e_{X}},
\end{aligned}
$$

where $\hat{e_{X}}$ is a unit vector perpendicular to the $Y$ axis on the surface of the electrodes. Here $\omega^{\prime}=\omega+2 \pi V / \lambda$ may be regarded as an equivalent angular frequency, with $\lambda$ the length scale of the imposed periodic spatial field along the $Y$ direction. $\lambda$ equals four times the sum of the electrode width and spacing in the present case. The translating velocity of the particle here may be generated by the traveling wave dielectrophoretic force or other microfluidic means, such as a pump. By substituting Eq. (12) into Eq. (7), and carrying out the integration, we found the long time response of the magnitude of the effective moment as

to AIP license or copyright; see http://apl.aip.org/apl/copyright.jsp 

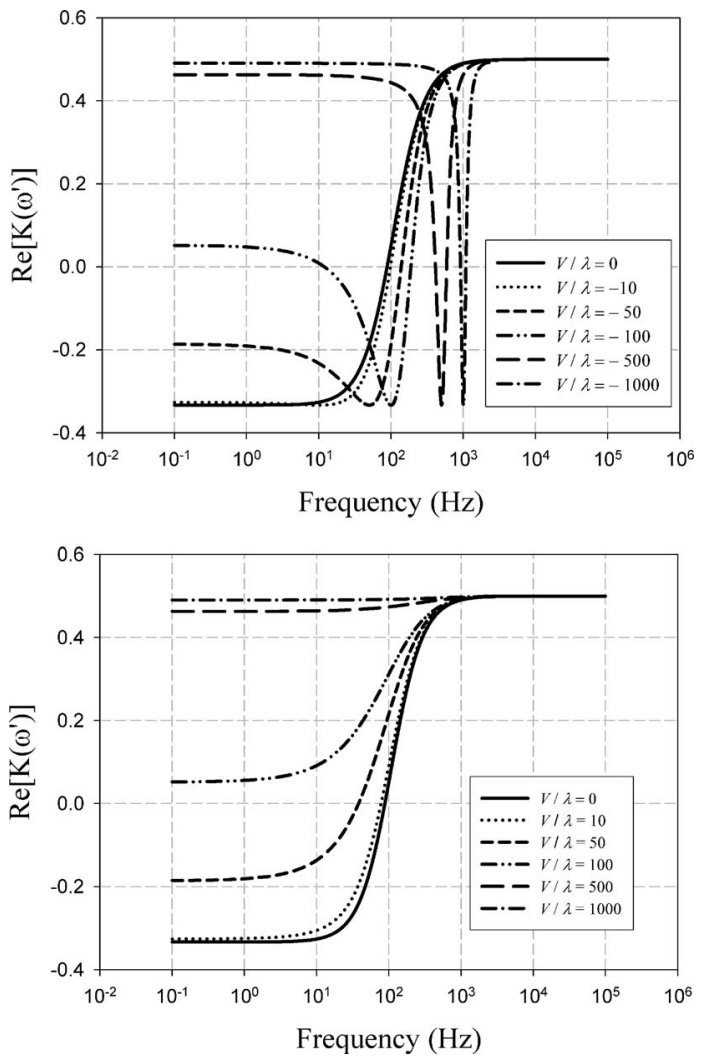

FIG. 1. Variation of $\operatorname{Re}\left[K\left(\omega^{\prime}\right)\right]$ with frequency $\omega$ for different $V / \lambda$.

$$
\begin{aligned}
m_{x}(t)= & 4 \pi \varepsilon_{1} R^{3} E_{0}\left\{\cos \left(\omega^{\prime} t\right) \operatorname{Re}\left[K\left(\omega^{\prime}\right)\right]\right. \\
& \left.-\sin \left(\omega^{\prime} t\right) \operatorname{Im}\left[K\left(\omega^{\prime}\right)\right]\right\},
\end{aligned}
$$

where $\operatorname{Re}\left[K\left(\omega^{\prime}\right)\right]$ and $\operatorname{Im}\left[K\left(\omega^{\prime}\right)\right]$ are the real and imaginary parts of the Clausius-Mossotti factor as calculated from Eqs. (11a) and (11b), but with $\omega$ replaced by $\omega^{\prime}$. On the other hand, if we calculate the magnitude of the effective moment using only the instantaneous local field (without considering the crossing trajectory effect), i.e., by substituting Eq. (12) directly into Eq. (10), we found

$$
\begin{aligned}
m_{x}(t)= & 4 \pi \varepsilon_{1} R^{3} E_{0}\left\{\cos \left(\omega^{\prime} t\right) \operatorname{Re}[K(\omega)]\right. \\
& \left.-\sin \left(\omega^{\prime} t\right) \operatorname{Im}[K(\omega)]\right\} .
\end{aligned}
$$

The difference between Eqs. (13) and (14) implies that the crossing trajectory effect on the present example is equivalent to a frequency shift effect on the Clausius-Mossotti factor for a translating particle. The frequency shift may be positive or negative depending on the sign of $V$. Whether this frequency shift effect is significant depends on the magnitude of $\omega /(2 \pi V / \lambda)$. For a typical cell manipulation ${ }^{7}$ using dielectrophoresis, $\lambda \approx 100 \mu \mathrm{m}$ and $V \approx 10-100 \mu \mathrm{m} / \mathrm{s}$ (may up to $1000 \mu \mathrm{m} / \mathrm{s}$ near the tips of the electrodes). In case the particle is further dragged by the fluid flow driven by a micropump, $V$ may reach or even exceed the order of $0.1 \mathrm{~m} / \mathrm{s}$. Consider again the case in Ref. 1 (page 41), $\operatorname{Re}\left[K\left(\omega^{\prime}\right)\right]$ and $\operatorname{Im}\left[K\left(\omega^{\prime}\right)\right]$ were calculated and plotted in Figs. 1 and 2, respectively. The results show that the frequency shift effect is negligible when $|V / \lambda|$ is sufficiently small and when $|\omega /(2 \pi V / \lambda)|>10$ roughly. For small $\omega$, both $\operatorname{Re}\left[K\left(\omega^{\prime}\right)\right]$ and $\operatorname{Im}\left[K\left(\omega^{\prime}\right)\right]$ change significantly as $|V / \lambda|$ increases. $\operatorname{Re}\left[K\left(\omega^{\prime}\right)\right]$ even reverses its sign when $|V / \lambda|$ is sufficiently large, which implies that negative dielectrophoresis may switch to positive dielectrophoresis or vice versa. The varia-
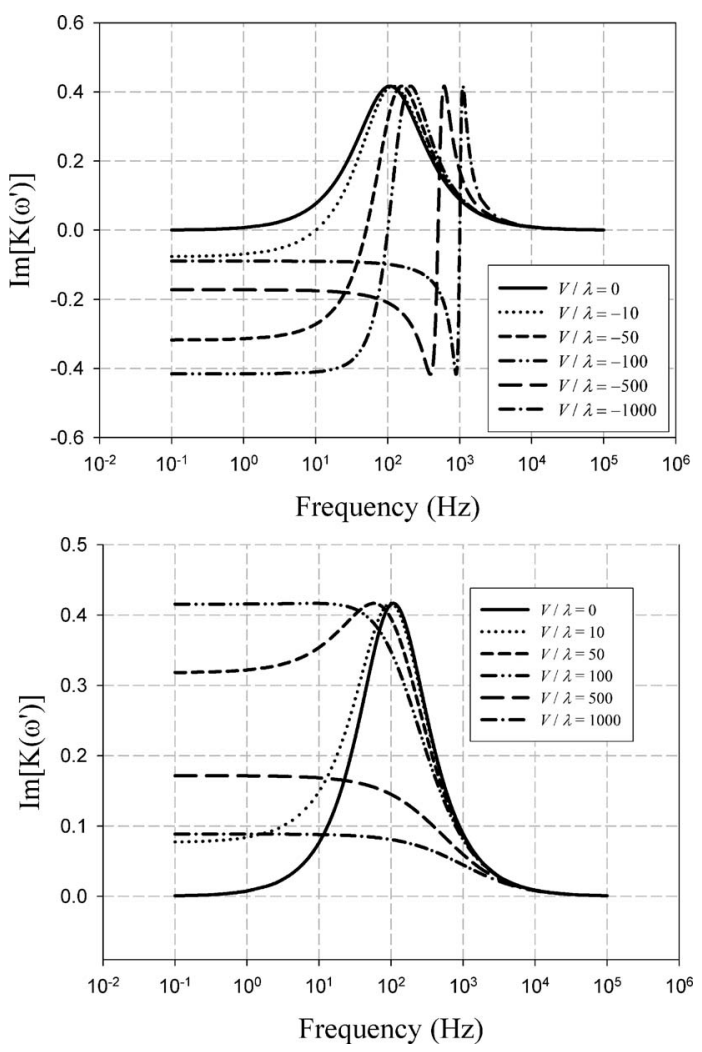

FIG. 2. Variation of $\operatorname{Im}\left[K\left(\omega^{\prime}\right)\right]$ with frequency $\omega$ for different $V / \lambda$.

tion of $\operatorname{Im}\left[K\left(\omega^{\prime}\right)\right]$ with $|V / \lambda|$ is not monotonic. The frequency range for nonzero $\operatorname{Im}\left[K\left(\omega^{\prime}\right)\right]$ on the low frequency side increases as $|V / \lambda|$ increases, which indicates that the traveling wave dielectrophoresis could be possible for a wider range of operation frequency. Also the peak of $\operatorname{Im}\left[K\left(\omega^{\prime}\right)\right]$ shifts continuously as $|V / \lambda|$ increases for negative $V / \lambda$. Figures 1 and 2 are based on $\lambda \approx 100 \mu \mathrm{m}$. The frequency range for the crossing trajectory effect to be important increases as $\lambda$ decreases, which implies that such effect could become more pronounced for the manipulation of micron and submicron particles. The above results presented in Figs. 1 and 2 are essential for illustrating the physics of crossing trajectory effect. Actually, Eq. (13) applies only to a uniformly translating spherical particle along the centerline of the channel in Ref. 7, where the electric field experienced by the particle can be described by Eq. (12) and the torque on the particle is identically zero. For cases in general, the problem should be analyzed by solving the equations of motion of the particle incorporated with Eqs. (8) and (9).

This work is supported partially by the National Science Council (NSC94-2120-M-002-014) and the Department of Economic Affair (94-EC-17-A-05-S1-017) of Taiwan, Republic of China.

${ }^{1}$ T. B. Jones, Electromechanics of Particles (Cambridge University Press, Cambridge, 1995).

${ }^{2}$ M. P. Hughes, Nanotechnology 11, 124 (2000).

${ }^{3}$ G. B. Denegri, G. Liberti, G. Molinari, and A. Viviani, IEEE Trans. Electr. Insul. EI-12, 114 (1977).

${ }^{4}$ G. Molinari and A. Viviani, J. Electrost. 5, 343 (1978).

${ }^{5}$ J. R. Melcher, Continuum Electromechanics (MIT, Cambridge, MA, 1982).

${ }^{6}$ M. R. Maxey and Riley, Phys. Fluids 26, 883 (1983).

${ }^{7}$ Y. Huang, X.-B. Wang, J. A. Tame, and R. Pethig, J. Phys. D 26, 1528 (1993). 